\title{
A Survey on Case-based Reasoning in Medicine
}

\author{
Nabanita Choudhury \\ Department of Computer Science \\ Assam University \\ Silchar, India
}

\author{
Shahin Ara Begum \\ Department of Computer Science \\ Assam University \\ Silchar, India
}

\begin{abstract}
Case-based reasoning (CBR) based on the memorycentered cognitive model is a strategy that focuses on how people learn a new skill or how they generate hypothesis on new situations based on their past experiences. Among various Artificial Intelligence tracks, CBR, due to its intrinsic similarity with the human reasoning process has been very promising in the utilization of intelligent systems in various domains, in particular in the domain of medicine. In this paper, we extensively survey the literature on CBR systems that are used in the medical domain over the past few decades. We also discuss the difficulties of implementing CBR in medicine and outline opportunities for future work.
\end{abstract}

Keywords-case-based reasoning; medicine; artificial intelligence; soft computing

\section{INTRODUCTION}

Case-Based Reasoning (CBR) is an area of machine learning research based on the memory-centered cognitive model [1]. CBR arose out of the research in cognitive science. It is defined as a model of reasoning that integrates problem solving, understanding and learning, and incorporates all of them with memory processes. It involves adapting earlier solutions to meet new demands, using old cases to explain or justify new solutions, and reasoning from past events to interpret a new situation. In CBR terminology, a case usually denotes a problem situation [2]. CBR can be considered as a form of similarity-based or analogical reasoning since the basic principle that is implicitly assumed to be applied in problem solving methodology is that similar problems have similar solutions [3].

CBR as a problem solving paradigm, is essentially different from other major Artificial Intelligence (AI) approaches in many aspects. Unlike other approaches which rely solely on the general knowledge of a problem domain, or which associate along inferred relationships between problem descriptors and conclusions, CBR utilizes the specific knowledge of previously experienced problem situations [2]. CBR can be applied as 'reasoning by experience in AI' as compared to rule-based reasoning which is applied as 'reasoning by logic in AI' [4]. The intuitive appeal of CBR comes due to its similarity to human problem solving behavior. Just as people draw on past experiences while solving a new problem, which often does not require in-depth analysis of the problem domain, CBR can be based on shallow knowledge and does not require significant effort in knowledge engineering as required by other AI fields like rule-based reasoning [5].

Medical reasoning on the other hand, involves processes 'that can be systematically analyzed, as well as those characterized as intangible' [6]. In medicine, the experts not only use rules to diagnose a problem, but they also use a mixture of textbook knowledge and experience. The experience consists of cases, typical and exceptional ones, and the physicians take them into account for reasoning. So, caseoriented methods should be very efficient in the domain of medical diagnosis, mainly because reasoning with cases corresponds with the typical decision making process of physicians. Also, incorporating new cases means automatically updating parts of the changeable knowledge [7]. Despite these, CBR has not become as successful in the medical domain, as it is in other fields for building intelligent systems [8].

The present paper surveys the available literature on systems developed using CBR for solving various problems in medicine. We begin in Section 2 by describing the basic notions of CBR and its models, with a brief description of the phases in CBR life cycle. Section 3 gives a brief description of medical reasoning. Section 4 surveys various CBR based systems developed over past few decades in the domain of medicine. In Section 5, we point out certain issues of using CBR in the field of medicine. Section 6 concludes the paper with a discussion on future directions of research.

\section{INSIDE CASE-BASED REASONING}

CBR is an analogical reasoning method, which means that it reasons from old cases or experiences to solve problems or interpret anomalous situations [9]. But the major difference between $\mathrm{CBR}$ and analogy is that analogy reasons across domains, whereas CBR reasons inside one domain [10]. In $\mathrm{CBR}$, the reasoning is based on remembering past experiences, as explained by Althoff et al. [11] - 'To solve a problem, remember a similar problem you have solved in the past and adapt the old solution to solve the new problem.' CBR can be interpreted in many ways [12] by different groups of people. For example, for cognitive scientists, it is a plausible high-level model for cognitive processing; for artificial intelligence researchers, it is a computational paradigm for solving problems; and for expert system practitioners, it is a design model.

CBR arose out of the research in cognitive science. The earliest contributions in this area were from Roger Schank and his colleagues at Yale University [2]. During the period 19771993, CBR research was regarded as a plausible high-level model for cognitive processing. Three CBR workshops were organized in 1988, 1989, and 1991 by the U.S. Defense Advanced Research Projects Agency (DARPA), which officially marked the birth of the discipline of CBR. In 1993, the first European workshop on CBR (EWCBR-93) was held 
in Kaiserslautern, Germany; and the first International Conference on CBR (ICCBR-95) was held in Sesimbra, Portugal. Many international workshops and conferences on CBR have been held in different parts of the world since then. Medical applications have been a part of the CBR community from the very beginning and are included in almost every international conference on CBR [13].

\section{A. CBR Models}

To understand the working of CBR, various models have been proposed in the literature. These include Hunt's model, Allen's model, Kolodner and Leake's model [14], and $\mathrm{R}^{4}$ model, developed by Aamodt \& Plaza [2]. Of these, the most widely used model and at the highest level of generality is the $\mathrm{R}^{4}$ model [15]. The process involved this model can be represented by a schematic cycle comprising of the four R's, as illustrated in Figure 1.

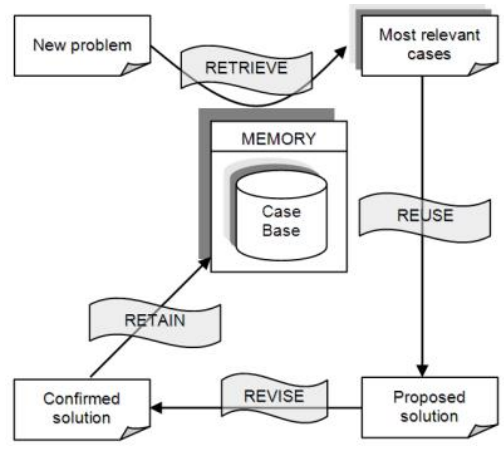

Fig. 1. The R4 Cycle [2]

- Retrieve the most similar case (s)

- Reuse the information and knowledge from retrieved case (s) to solve the problem

- Revise the proposed solution

- Retain the parts of this solution likely to be useful in future.

\section{B. CBR Life Cycle}

The problem solving life cycle of CBR essentially consists of retrieval, adaptation, and maintenance. Each of these has its own importance in the successful working of the CBR system.

1) Retrieval

Retrieval is often considered the most important phase of CBR since it lays the foundation for overall working of the CBR system [16]. Retrieval includes the process of finding those cases within a case base, which are most similar to the current case. The most commonly investigated retrieval techniques include nearest neighbor retrieval, inductive approaches, knowledge guided approaches, and validated retrieval [5], [17]. Some hybrid algorithms have also been proposed e.g. Discretised Highest Similarity with Pattern Solution Re-use algorithm [18].

\section{2) Adaptation}

The next two phases of the CBR cycle, viz. reuse and revise are often difficult to distinguish in many practical applications, as a result of which many researchers replace and combine them into a single stage called adaptation [5]. In the early 90's the CBR community focused on retrieval only. Investigations of the various aspects of adaptation started after that [19]. Most of the advances also have been achieved at the retrieval and retain phase of CBR [20]. In the reuse phase, advances have been obtained depending on the system purpose viz. diagnosis, classification, tutoring and planning (such as therapy support). Regarding diagnosis and classification, most of the systems rely on adaptation methods that consist of copying the solution of the most similar case or a combination of them, i.e. reusing the solution [21].

\section{3) Maintenance}

After reusing and revising the retrieved case, the next step in CBR cycle is to retain the case (s). There are many approaches to achieve this. Many systems store only the solution of the previous problem, whereas some systems store the solving process [16]. In many cases, this process of retaining leads to an uncontrolled growth in the case base, which in turn leads to a poorer performance of the system in terms of speed [22]. So, the need of maintaining a case base arises.

\section{MEDICAL REASONING}

Medical reasoning is divided into diagnostic reasoning, planning, and patient management [23]. This reasoning is carried out in terms of physiological states, complaints, symptoms and so forth [24]. Diagnostic reasoning includes cognitive activities like gathering information, recognition of patterns, solving problems and decision making [25]. Diagnostic investigations are quite complex and error prone [26]. Table 1 outlines the diagnostic process.

\section{TABLE I. DiAgNostic CYCLE [27]}

\begin{tabular}{|l|l|}
\hline Step & Decision \\
\hline 1 & Select a diagnostic test (or question) \\
\hline 2 & Carry out the selected test and observe its outcome \\
\hline 3 & $\begin{array}{l}\text { Either (i) select a further diagnostic test and so return to step 1; } \\
\text { or (ii) make a diagnosis in the light of the outcomes so far } \\
\text { obtained }\end{array}$ \\
\hline
\end{tabular}

This diagnosis process may become easier and more reliable if equipped with an expert system that provides past diagnosis of cases, thereby helping the physician to arrive at a solution based on the past experiences [28].

\section{CBR SYSTEMS IN MEDICINE}

CBR used in medical reasoning literature is termed as 'instance-based recognition' [29]. Unlike other knowledge domains, cases have to be professionally documented in medical domain [30]. The very fact that the methodology of CBR systems closely resembles the thought process of a physician suggests a successful use of CBR in medicine [31]. Koton pointed out while introducing CASEY - 'A physician's problem-solving performance improves with experience. The performance of most medical expert systems does not' [32]. The experts in the medical domain do not use rules for diagnosis. What they use is the knowledge they obtain from books, as well as experiences just the way in which CBR works [7]. 
The main advantage of CBR systems in medicine is the automatic formation of a facility adapted knowledge base [33], which is a very important aspect in medical decision making. Also, the continuously changing nature of medical knowledge base, presence of more than one solution, and complexity in modeling also make CBR applicable in medical domain [34]. As a result, CBR has been used for building intelligent computer-aided decision support systems in the medical domain in the past few decades [35].
CBR decision support systems can be classified [20] as planning, classification, tutoring, and diagnostic systems based on their purpose oriented properties. Table 2 lists in chronological order, some of the CBR systems developed in the field of medical reasoning over the years. Also, it classifies these systems according to their objectives and attempts to find out the extent to which adaptation phase of CBR is used in these systems.

TABLE II. CBR SYSTEMS IN MEDICINE

\begin{tabular}{|c|c|c|c|c|c|c|}
\hline Author(s) & System & Ref. & Objective & Technique(s) used & $\begin{array}{c}\text { Area of } \\
\text { Application }\end{array}$ & Adaptation (if any) \\
\hline Koton & CASEY & [32] & Diagnosis & $\begin{array}{l}\text { CBR, Rule-based domain } \\
\text { theory, and Model-based } \\
\text { reasoning }\end{array}$ & $\begin{array}{l}\text { Coronary } \\
\text { disease }\end{array}$ & $\begin{array}{l}\text { Adaptation with rules } \\
\text { attempted }\end{array}$ \\
\hline $\begin{array}{c}\text { Bareiss, Porter \& } \\
\text { Wier } \\
\end{array}$ & Protos & [36] & $\begin{array}{c}\text { Classification and } \\
\text { Diagnosis } \\
\end{array}$ & 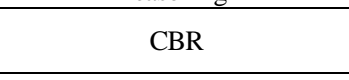 & $\begin{array}{l}\text { Hearing } \\
\text { disorders }\end{array}$ & No adaptation \\
\hline $\begin{array}{l}\text { Gierl \& Stengel- } \\
\text { Rutkowski }\end{array}$ & GS.52 & [37] & Diagnosis & CBR & $\begin{array}{l}\text { Dysmorphic } \\
\text { syndromes }\end{array}$ & $\begin{array}{l}\text { Adaptation performed with } \\
\text { the application of constraints } \\
\text { (contradictions) }\end{array}$ \\
\hline Macura \& Macura & MacRad & [38] & Classification & CBR & $\begin{array}{l}\text { Radiology } \\
\text { Images }\end{array}$ & No adaptation \\
\hline $\begin{array}{l}\text { Haddad, Moertl \& } \\
\text { Porenta }\end{array}$ & SCINA & [39] & $\begin{array}{c}\text { Image } \\
\text { Interpretation }\end{array}$ & CBR and Rule-based reasoning & $\begin{array}{c}\text { Myocardial } \\
\text { Perfusion } \\
\text { Scintigrams } \\
\end{array}$ & $\begin{array}{c}\text { Adaptation performed with } \\
\text { Rule-base }\end{array}$ \\
\hline $\begin{array}{c}\text { Reategui, } \\
\text { Campbell, \& Leao }\end{array}$ & -- & [40] & Diagnosis & CBR and Neural networks & $\begin{array}{l}\text { Congenital heart } \\
\text { diseases }\end{array}$ & No adaptation \\
\hline Hsu \& Ho & -- & [28] & Diagnosis & $\begin{array}{l}\text { CBR, Fuzzy logic, Neural } \\
\text { networks, Induction, and } \\
\text { Knowledge-based technology }\end{array}$ & General & $\begin{array}{c}\text { Adaptation performed with } \\
\text { Rule-base }\end{array}$ \\
\hline $\begin{array}{c}\text { Bichindaritz, Kansu } \\
\text { \& Sullivan }\end{array}$ & CARE-PARTNER & [41] & $\begin{array}{c}\text { Knowledge- } \\
\text { support assistance }\end{array}$ & $\begin{array}{l}\text { CBR, Rule-based reasoning, } \\
\text { and Information retrieval }\end{array}$ & General & $\begin{array}{l}\text { Adaptation performed with } \\
\text { rules, cases and pathways }\end{array}$ \\
\hline LeBozec et al. & IDEM & [42] & Classification & CBR & $\begin{array}{l}\text { Radiology } \\
\text { Images }\end{array}$ & No Adaptation \\
\hline $\begin{array}{l}\text { Gierl, Bull, \& } \\
\text { Schmidt }\end{array}$ & TeCoMED & [30] & $\begin{array}{l}\begin{array}{c}\text { Classification } \\
\text { (forecasting) }\end{array} \\
\end{array}$ & $\begin{array}{l}\text { CBR, Rule-based reasoning, } \\
\text { and Model-based reasoning }\end{array}$ & Epidemics & Compositional Adaptation \\
\hline Perner & -- & [43] & $\begin{array}{l}\text { Classification, } \\
\text { Knowledge } \\
\text { acquisition/ } \\
\text { management }\end{array}$ & $\begin{array}{l}\text { CBR, Image processing, and } \\
\text { Data mining }\end{array}$ & $\begin{array}{l}\text { Medical image } \\
\text { analysis }\end{array}$ & No Adaptation \\
\hline $\begin{array}{c}\text { Schmidt, Pollwein, } \\
\text { \& Gierl }\end{array}$ & COSYL & [44] & Classification & CBR & $\begin{array}{c}\text { Liver } \\
\text { transplantation }\end{array}$ & No Adaptation \\
\hline $\begin{array}{c}\text { Goodridge, Peter, } \\
\text { \&Abayomi }\end{array}$ & MED2000 & [45] & Diagnosis & CBR and Neural networks & $\begin{array}{c}\text { Hematological } \\
\text { diseases }\end{array}$ & No Adaptation \\
\hline $\begin{array}{l}\text { Phuong, Thang, \& } \\
\text { Hirota }\end{array}$ & -- & [46] & Diagnosis & CBR and Fuzzy logic & Lung diseases & No Adaptation \\
\hline $\begin{array}{c}\text { Marling \& } \\
\text { Whitehouse }\end{array}$ & Auguste & [47] & Planning & CBR and Rule-based reasoning & $\begin{array}{l}\text { Alzheimer's } \\
\text { disease }\end{array}$ & No Adaptation \\
\hline Golobardes et al. & $\mathrm{CaB}-\mathrm{CS}$ & [48] & Classification & CBR & Breast cancer & No Adaptation \\
\hline Montani et al. & -- & [49] & Planning & $\begin{array}{l}\text { CBR, Rule-based reasoning, } \\
\text { and Model-based reasoning }\end{array}$ & Type 1 diabetes & $\begin{array}{c}\text { Adaptation performed with } \\
\text { rules }\end{array}$ \\
\hline $\begin{array}{l}\text { Vorobieva, Gierl, } \\
\text { \& Schmidt }\end{array}$ & -- & {$[50]$} & Planning & CBR & Endocrinology & $\begin{array}{l}\text { Adaptation performed and } \\
\text { task oriented adaptation } \\
\text { model developed }\end{array}$ \\
\hline Hsu \& Ho & -- & {$[51]$} & Diagnosis & $\begin{array}{l}\text { CBR, Neural networks, Fuzzy } \\
\text { theory, Induction, Utility } \\
\text { theory, and Knowledge-based } \\
\text { planning technology }\end{array}$ & $\begin{array}{l}\text { Multiple } \\
\text { diseases }\end{array}$ & $\begin{array}{l}\text { Adaptation performed with } \\
\text { knowledge-based planning }\end{array}$ \\
\hline Nilsson \& Funk & -- & {$[52]$} & Classification & CBR and Rule-based reasoning & $\begin{array}{c}\text { Respiratory } \\
\text { sinus arrhythmia }\end{array}$ & No Adaptation \\
\hline $\begin{array}{c}\text { Kwiatkowska \& } \\
\text { Atkins }\end{array}$ & Somnus & {$[53]$} & Diagnosis & $\begin{array}{l}\text { CBR, Fuzzy logic, and } \\
\text { Semiotics }\end{array}$ & $\begin{array}{l}\text { Obstructive } \\
\text { sleep apnea }\end{array}$ & No Adaptation \\
\hline Perner et al. & -- & {$[54]$} & $\begin{array}{l}\text { Classification, } \\
\text { Knowledge } \\
\text { acquisition/ } \\
\text { management }\end{array}$ & $\mathrm{CBR}$ and Image processing & $\begin{array}{l}\text { Recognition of } \\
\text { Airborne Fungi } \\
\quad \text { Spores }\end{array}$ & No Adaptation \\
\hline
\end{tabular}




\begin{tabular}{|c|c|c|c|c|c|c|}
\hline $\begin{array}{c}\text { Brien, Glasgow \& } \\
\text { Munoz }\end{array}$ & -- & {$[55]$} & Classification & CBR & $\begin{array}{l}\text { Attention-deficit } \\
\text { hyperactivity } \\
\text { disorder }\end{array}$ & No Specific Adaptation \\
\hline Chang & -- & {$[56]$} & Diagnosis & CBR & $\begin{array}{c}\text { Development } \\
\text { delay in children }\end{array}$ & $\begin{array}{l}\text { Adaptation performed with } \\
\text { the help of human experts }\end{array}$ \\
\hline Shi \& Barnden & -- & {$[57]$} & Diagnosis & $\mathrm{CBR}$ and Induction & $\begin{array}{l}\text { Multiple } \\
\text { disorders }\end{array}$ & $\begin{array}{l}\text { Abductive Adaptation with } \\
\text { rules }\end{array}$ \\
\hline $\begin{array}{l}\text { Montani \& } \\
\text { Portinale }\end{array}$ & RHENE & {$[58]$} & $\begin{array}{l}\text { Classification, } \\
\text { planning, } \\
\text { knowledge } \\
\text { acquisition/ } \\
\text { management }\end{array}$ & $\begin{array}{l}\text { CBR and Temporal } \\
\text { Abstractions }\end{array}$ & Hemodialysis & No Adaptation \\
\hline $\begin{array}{l}\text { d'Aquin, Lieber \& } \\
\text { Napoli }\end{array}$ & KASIMIR & [59] & $\begin{array}{l}\text { Diagnosis, } \\
\text { classification, } \\
\text { knowledge } \\
\text { acquisition/ } \\
\text { management }\end{array}$ & $\begin{array}{l}\text { CBR, Semantic web, Belief } \\
\text { revision theory, Fuzzy logic, } \\
\text { and Ergonomy }\end{array}$ & Breast cancer & $\begin{array}{c}\text { Adaptation Performed } \\
\text { (Adaptation Guided } \\
\text { Retrieval) }\end{array}$ \\
\hline $\begin{array}{l}\text { Díaz, Fdez- } \\
\text { Riverola \& } \\
\text { Corchado } \\
\end{array}$ & geneCBR & {$[60]$} & $\begin{array}{l}\text { Diagnosis and } \\
\text { classification }\end{array}$ & CBR and Fuzzy Logic & Cancer & $\begin{array}{l}\text { Adaptation Performed with } \\
\text { the help of human expert }\end{array}$ \\
\hline Park, Kim \& Chun & -- & {$[61]$} & $\begin{array}{c}\text { Classification and } \\
\text { diagnosis }\end{array}$ & CBR and Probability & General & No Adaptation \\
\hline $\begin{array}{l}\text { Töpel, Neumann \& } \\
\text { Hofestadt }\end{array}$ & -- & {$[62]$} & $\begin{array}{l}\text { Diagnosis and } \\
\text { planning therapy } \\
\text { information }\end{array}$ & CBR & $\begin{array}{l}\text { Inborn metabolic } \\
\text { disease }\end{array}$ & No Adaptation \\
\hline Quellec et al. & -- & {$[63]$} & Classification & $\mathrm{CBR}$ and Decision trees & $\begin{array}{l}\text { Diabetic } \\
\text { retinopathy }\end{array}$ & No Adaptation \\
\hline Cordier et al. & FrakaS & {$[64]$} & $\begin{array}{l}\text { Diagnosis, } \\
\text { Knowledge } \\
\text { acquisition/ } \\
\text { management }\end{array}$ & CBR & Oncology & $\begin{array}{c}\text { Conservative adaptation } \\
\text { performed }\end{array}$ \\
\hline $\begin{array}{l}\text { Marling, Shubrook } \\
\text { \& Schwartz }\end{array}$ & -- & {$[65]$} & Planning & CBR & Type 1 diabetes & No adaptation \\
\hline $\begin{array}{l}\text { Little, Salvetti \& } \\
\text { Perner }\end{array}$ & ProtoClass & {$[66]$} & Classification & CBR & General & No adaptation \\
\hline Ahmed et al. & -- & {$[67]$} & Diagnosis & CBR and Fuzzy logic & Stress & No adaptation \\
\hline Rodríguez et al. & SAPRIM & {$[68]$} & Prediction & $\begin{array}{c}\text { CBR, Neural networks, and } \\
\text { Fuzzy Logic }\end{array}$ & Pediatric risk & No adaptation \\
\hline $\begin{array}{l}\text { Corchado, Bajo \& } \\
\text { Abraham }\end{array}$ & GerAmi & [69] & $\begin{array}{c}\text { Planning, } \\
\text { Knowledge } \\
\text { acquisition/ } \\
\text { management } \\
\end{array}$ & $\mathrm{CBR}$ and Variational calculus & $\begin{array}{l}\text { Alzheimer's } \\
\text { disease }\end{array}$ & Adaptation performed \\
\hline De Paz et al. & -- & {$[70]$} & $\begin{array}{c}\text { Diagnosis and } \\
\text { classification }\end{array}$ & $\begin{array}{c}\text { CBR, Neural networks, and } \\
\text { Statistics } \\
\end{array}$ & Leukemia & $\begin{array}{c}\text { Adaptation performed with } \\
\text { Classification Tree }\end{array}$ \\
\hline Obot \& Uzoka & -- & [71] & Diagnosis & $\begin{array}{l}\text { CBR, Rule-based reasoning, } \\
\text { and Neural networks }\end{array}$ & Hepatitis & $\begin{array}{c}\text { Adaptation performed with } \\
\text { the help of rules and neural } \\
\text { network }\end{array}$ \\
\hline Lin & -- & [72] & Diagnosis & $\begin{array}{l}\text { CBR and Classification and } \\
\text { regression tree (CART) }\end{array}$ & Liver diseases & No adaptation \\
\hline Ahn \& Kim & GOCBR & [73] & Diagnosis & CBR and Genetic algorithms & Breast Cancer & No adaptation \\
\hline Begum et al. & -- & {$[74]$} & Diagnosis & CBR and Fuzzy logic & Stress & No adaptation \\
\hline Yao \& Li & ANMM4CBR & {$[75]$} & Classification & CBR & $\begin{array}{c}\text { Gene expression } \\
\text { data }\end{array}$ & No adaptation \\
\hline Gu et al. & CBR-DENT & [76] & $\begin{array}{l}\text { Knowledge } \\
\text { management }\end{array}$ & CBR and Fuzzy Logic & Odontology & Adaptation performed \\
\hline Lin \& Chuang & -- & [77] & Diagnosis & $\begin{array}{l}\text { CBR, Analytic hierarchy } \\
\text { process, and Neural networks }\end{array}$ & Liver diseases & No adaptation \\
\hline Jagannathan et al. & -- & {$[78]$} & Planning & CBR and Fuzzy logic & $\begin{array}{l}\text { Brain cancer } \\
\text { radiotherapy }\end{array}$ & $\begin{array}{c}\text { Adaptation suggested, but } \\
\text { not performed }\end{array}$ \\
\hline Ahmed et al. & -- & [79] & Planning & CBR and Fuzzy logic & Stress & Adaptation performed \\
\hline Douali et al. & -- & {$[80]$} & Diagnosis & $\begin{array}{c}\text { Case-based Fuzzy cognitive } \\
\text { maps }\end{array}$ & $\begin{array}{l}\text { Urinary tract } \\
\text { infection }\end{array}$ & No adaptation \\
\hline Chuang & -- & {$[81]$} & Diagnosis & $\begin{array}{l}\text { CBR and Neural networks } \\
\text { (Back propagation network) }\end{array}$ & Liver disease & No adaptation \\
\hline $\begin{array}{c}\text { Petrovic, Mishra \& } \\
\text { Sundar }\end{array}$ & -- & {$[82]$} & Planning & $\begin{array}{l}\text { CBR and Dempster-Shafer } \\
\text { theory }\end{array}$ & Prostate Cancer & Adaptation performed \\
\hline $\begin{array}{l}\text { van den Branden et } \\
\text { al. }\end{array}$ & $\begin{array}{l}\text { Excelicare } \\
\text { CBR }\end{array}$ & [83] & Classification & CBR and Genetic Algorithm & $\begin{array}{c}\text { Electronic } \\
\text { patient record }\end{array}$ & No adaptation \\
\hline
\end{tabular}




\begin{tabular}{|c|c|c|c|c|c|c|}
\hline López et al. & $\mathrm{eXiT} * \mathrm{CBR}$ & $\begin{array}{l}{[84],} \\
{[85]}\end{array}$ & Diagnosis & $\begin{array}{l}\text { CBR, Pedigree tools, and } \\
\text { Genetic algorithms }\end{array}$ & Breast cancer & Adaptation performed \\
\hline $\begin{array}{l}\text { Ahmed, Begum \& } \\
\text { Funk }\end{array}$ & -- & [86] & Diagnosis & $\begin{array}{l}\text { CBR, Fuzzy logic, Rule-based } \\
\text { reasoning, and Textual } \\
\text { information retrieval }\end{array}$ & Stress & No adaptation \\
\hline Montani et al. & -- & [87] & $\begin{array}{l}\text { Classification and } \\
\text { planning }\end{array}$ & CBR & Hemodialysis & No adaptation \\
\hline Khelassi et al. & -- & [88] & Diagnosis & $\begin{array}{l}\text { CBR, Rule-based reasoning, } \\
\text { Distributed reasoning, and } \\
\text { Fuzzy logic }\end{array}$ & $\begin{array}{l}\text { Cardiac } \\
\text { arrhythmia }\end{array}$ & No adaptation \\
\hline Marling et al. & 4DSS & [89] & Planning & CBR and Rule-based reasoning & Type 1 diabetes & Adaptation performed \\
\hline Juarez et al. & GRACE & [90] & $\begin{array}{c}\text { Supporting } \\
\text { protocol design }\end{array}$ & CBR & $\begin{array}{c}\text { Frontotemporal } \\
\text { dementia }\end{array}$ & $\begin{array}{l}\text { Adaptation performed with } \\
\text { rule-base }\end{array}$ \\
\hline $\begin{array}{l}\text { Ahmed, Begum, \& } \\
\text { Funk }\end{array}$ & -- & [91] & $\begin{array}{l}\text { Diagnosis, } \\
\text { classification and } \\
\text { planning }\end{array}$ & $\begin{array}{l}\text { CBR, Fuzzy logic, Rule-based } \\
\text { reasoning, and Textual } \\
\text { information retrieval }\end{array}$ & $\begin{array}{c}\text { Stress } \\
\text { Management }\end{array}$ & No adaptation \\
\hline $\begin{array}{l}\text { Ahmed, Islam, \& } \\
\text { Loutfi }\end{array}$ & -- & [92] & $\begin{array}{c}\text { Patient } \\
\text { identification }\end{array}$ & CBR & General & No adaptation \\
\hline $\begin{array}{c}\text { Begum, Ahmed, \& } \\
\text { Barua }\end{array}$ & - & [93] & Classification & CBR and Fuzzy logic & $\begin{array}{l}\text { Physiological } \\
\text { sensor signals }\end{array}$ & No adaptation \\
\hline $\begin{array}{l}\text { Ekong, Inyang, \& } \\
\text { Onibere }\end{array}$ & -- & [94] & Diagnosis & $\begin{array}{l}\text { CBR, Neural networks, and } \\
\text { Fuzzy logic }\end{array}$ & $\begin{array}{c}\text { Depression } \\
\text { disorder }\end{array}$ & No adaptation \\
\hline Huang et al. & -- & [95] & $\begin{array}{l}\text { Classification and } \\
\text { diagnosis }\end{array}$ & $\begin{array}{l}\text { CBR, Neural networks, and } \\
\text { Adaptive Neuro-Fuzzy } \\
\text { Inference System }\end{array}$ & Breast cancer & No adaptation \\
\hline Montani et al. & -- & [96] & $\begin{array}{l}\text { Classification } \\
\text { (retrieval) }\end{array}$ & CBR & $\begin{array}{l}\text { Comparative } \\
\text { genomics }\end{array}$ & No adaptation \\
\hline $\begin{array}{c}\text { Chattopadhyay et } \\
\text { al. }\end{array}$ & -- & [97] & Diagnosis & CBR & $\begin{array}{l}\text { Premenstrual } \\
\text { syndrome }\end{array}$ & Adaptation \\
\hline Pla et al. & $\begin{array}{l}\mathrm{eXiT}^{*} \mathrm{CBR} . \\
\text { v2 }\end{array}$ & [98] & Diagnosis & $\begin{array}{l}\text { CBR, Genetic algorithms, and } \\
\text { Cooperative multi agent system } \\
\text { technology }\end{array}$ & General & Adaptation performed \\
\hline Leal et al. & -- & [99] & Planning & $\begin{array}{l}\text { CBR and Principal component } \\
\text { analysis }\end{array}$ & $\begin{array}{l}\text { Continuous } \\
\text { glucose } \\
\text { monitoring } \\
\text { systems in } \\
\text { intensive care } \\
\text { unit }\end{array}$ & No adaptation \\
\hline $\begin{array}{l}\text { Teodorović, } \\
\text { Šelmić, \& } \\
\text { Mijatović- } \\
\text { Teodorović }\end{array}$ & -- & [100] & Planning & $\begin{array}{l}\text { CBR and Bee colony } \\
\text { optimization }\end{array}$ & Thyroid cancer & No adaptation \\
\hline Henriet et al. & EquiVox & [101] & $\begin{array}{l}\text { Representations of } \\
\text { human organs }\end{array}$ & CBR and Neural networks & $\begin{array}{l}\text { Numerical } \\
\text { representation of } \\
\text { human organs }\end{array}$ & $\begin{array}{c}\text { Adaptation performed with } \\
\text { ANN }\end{array}$ \\
\hline Sharaf-El-Deen & -- & [102] & Diagnosis & CBR and Rule-based reasoning & $\begin{array}{l}\text { Breast Cancer } \\
\text { and Thyroid } \\
\text { disease }\end{array}$ & $\begin{array}{l}\text { Adaptation performed with } \\
\text { rules }\end{array}$ \\
\hline Yin et al. & -- & [103] & Diagnosis & CBR & Headache & No adaptation \\
\hline Tyagi \& Singh & -- & [104] & Classification & CBR & Asthma & No adaptation \\
\hline $\begin{array}{l}\text { Khussainova, } \\
\text { Petrovic, \& } \\
\text { Jagannathan }\end{array}$ & -- & [105] & Planning & CBR and Clustering & $\begin{array}{l}\text { Radiotherapy } \\
\text { (Brain Cancer) }\end{array}$ & No adaptation \\
\hline Saraiva et al. & -- & [106] & Diagnosis & CBR and Rule-based reasoning & $\begin{array}{c}\text { Four types of } \\
\text { gastrointestinal } \\
\text { cancer }\end{array}$ & No adaptation \\
\hline Chakraborty et al. & CEDS & [107] & Diagnosis & CBR & Cholera & No adaptation \\
\hline $\begin{array}{l}\text { Nasiri, Zenkert, \& } \\
\text { Fathi }\end{array}$ & DePicT & [108] & $\begin{array}{l}\text { Diagnosis and } \\
\text { recommendation }\end{array}$ & CBR & & No adaptation \\
\hline $\begin{array}{l}\text { Banerjee \& } \\
\text { Chowdhury }\end{array}$ & -- & [109] & Diagnosis & $\begin{array}{l}\text { CBR, Fuzzy clustering, and } \\
\text { Decision trees }\end{array}$ & $\begin{array}{c}\text { Retinal } \\
\text { Abnormalities }\end{array}$ & No adaptation \\
\hline
\end{tabular}

From our study, it was observed that CBR in the medical domain has a wide range of application. Most of the systems are developed specifically to deal with a particular disease. Secondly, most of the systems act as prototypes, and not as final products, as mentioned by Blanco [110]. These systems require a human expert to interpret the final result. Another visible trend was the successful hybridization of $\mathrm{CBR}$ with soft computing methods. 32 out of 76 systems studied by us have used some or the other soft computing techniques in addition to CBR. Moreover, among the 76 systems, 51 systems completely avoid automatic adaptation and mainly work as retrieval only systems. The other systems do have the adaptation phase in them, but often the reasoning mechanism in those is coupled with rule-based reasoning, or various soft computing methods. 


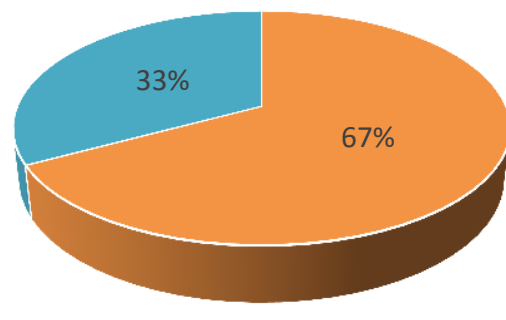

- No Adaptation - Adaptation

Fig. 2. Percentage of systems in terms of adaptation performed

\section{PROBLEMS IN MEDICAL CBR SYSTEMS}

Though the above discussion reflects the successful use of $\mathrm{CBR}$ in medicine, there are some limitations which restrict the use of CBR in medicine. In a medical case, the number of features is often extremely large, thereby making the generalization and adaptation quite difficult [20]. At the same time, reliability cannot be guaranteed in medical CBR systems [111]. The limited number of reference cases aids to the problem of implementing a medical CBR system [35]. But the most important concern in the successful implementation of medical CBR systems is the adaptation problem. As our study suggests, so far, the number of systems in the medical domain that apply the complete CBR method is very less. Most of the systems use no adaptation at all, and the task of adaptation is left to the human expert.

d'Aquin et al. [59] remark that adaptation in medicine is quite a complex procedure, as it needs to deal with the lack of relevant information about a patient, the applicability and consequences of the decision, the closeness to the decision thresholds and the necessity to consider patients according to different viewpoints. Schmidt et al. [7] also point out that giving autonomy to the adaptation step of CBR has been a difficult step in Medicine. Due to these challenges, most of the advances made in medical CBR systems focus on the retrieval phase. The adaptation phase is limited to planning tasks [21]. No general models have been developed for adaptation as it largely depends on the domain and application characteristics.

Our study reveals that medical CBR systems deal with the adaptation problem in two ways. Most of the systems avoid the adaptation problem by applying only retrieval phase of CBR cycle [19] while some others attempt to solve it. One of the earliest medical expert systems, CASEY [32] makes an attempt to solve the adaptation task. In this, the creation of a complete rule base for adaptation is time consuming, as a result of which a few general operators are used for adaptation. And when no similar case can be found or if adaptation fails, CASEY uses a rule-based domain theory. But since knowledge acquisition is the bottleneck for the development of rule-based medical expert systems, the development of complete adaptation rule bases have never become a successful technique to solve the adaptation problem in medical CBR systems [7]. The application of constraints leads to a better solution, as in the GS.52 project [37] but only for specific situations. KASIMIR [59] uses similarity paths and reformulation to support the adaptation, but adaptation knowledge in the form of rules is still required. Some of the more recent systems perform adaptation successfully, with the help of soft computing techniques, e.g. eXiT*CBR.v2 [98] revises and reuses the cases using genetic algorithms; EquiVox developed by Henriet et al. [101] performs adaptation using artificial neural networks. So, the inclusion of soft computing techniques suggests improved automatic adaptation in medical CBR systems.

\section{CONCLUSIONS AND FUTURE SCOPE}

A fundamental part of the CBR system is learning by remembering cases. CBR systems, cognitively similar to human beings, take into account previous experiences for solving new problems, consider both subjective and objective knowledge unlike other expert systems, and can incrementally acquire knowledge automatically, but still, these are not as successful in medicine as in other domains. The main reason for this is the adaptation problem. The retrieval and maintenance phases have gained a lot of attention of the researchers, while the adaptation phase is still in its infancy. The adaptation phase involves multifarious problems which include dealing with the closeness to the decision threshold used to determine similar cases, among other issues. The majority of the medical CBR systems avoid the adaptation problem, and act as retrieval only systems and leave case adaptation and case update to be performed by human experts. A solution to adaptation problem is the integration of CBR with other methodologies. The synergism of these methodologies leads to the development of new sophisticated and hybridized systems.

It was observed in our survey that a majority of successful medical CBR systems are built around a combination of CBR and other artificial intelligence methods. From the very beginning, hybrid systems came into existence for medical CBR systems; Koton's CASEY [32] being an example which hybridizes $\mathrm{CBR}$ and RBR. Soft computing techniques viz. fuzzy logic, artificial neural networks, in particular backpropagation neural networks and Bayesian models, and evolutionary strategies have proved to be very efficient in enhancing the capabilities of CBR systems. With the use of these techniques, adaptation knowledge can be determined automatically from the cases, which leads to more robustness of this knowledge [5]. Schmidt, Vorobieva, \& Gierl [8] have mentioned that the application of adaptation rules or operators, though general seems to be the only technique which can solve medical adaptation problems. We suggest the use of fuzzy decision trees for this; wherein fuzzy decision rules can be generated, and rough set techniques can be used to simplify these rules.

In the domain of medicine, where clear domain knowledge is often not available, automatic adaptation is difficult to develop. So, hybrid combinations of soft computing techniques may be explored and implemented in greater details in the adaptation phase of CBR to move forward the success story of CBR in the otherwise difficult domain of medicine.

\section{REFERENCES}

[1] L. D. Xu, "Case based reasoning," Potentials, IEEE, vol. 13, no. 5, pp. 10-13, 1994. 
[2] A. Aamodt and E. Plaza, "Case-based reasoning: Foundational issues, methodological variations, and system approaches," AI communications, vol. 7, no. 1, pp. 39-59, 1994.

[3] E. Armengol, F. Esteva, L. Godo, and Torra, "On learning similarity relations in fuzzy case-based reasoning," Transactions on Rough Sets II, Lecture Notes in Computer Science, vol. 3135, pp. 14-32, 2004.

[4] M. Ahmed, S. Begum, and P. Funk, "The 3 CDSSs: An overview and application in case-based reasoning," in Proceedings of the 27th annual workshop of the Swedish artificial intelligence society, pp. 25-32, 2012.

[5] S. K. Pal and S. C. Shiu, Foundations of soft case-based reasoning, John Wiley \& Sons, 2004.

[6] R. S. Ledley and L. B. Lusted, "Reasoning Foundations of Medical Diagnosis Symbolic logic, probability, and value theory aid our understanding of how physicians reason," Science, vol. 130, no. 3366, pp. 9-21, 1959.

[7] R. Schmidt, S. Montani, R. Bellazzi, L. Portinale, and L. Gierl, "Casedbased reasoning for medical knowledge-based systems," International Journal of Medical Informatics, vol. 64, no. 2, pp. 355-367, 2001.

[8] R. Schmidt, O. Vorobieva, and L. Gierl, "Case-based adaptation problems in medicine," in Proceedings of WM2003: Professionelles Wissensmanagement, Kollen-Verlag Bonn, 2003.

[9] J. L. Kolodner, "Improving human decision making through case-based decision aiding. AI magazine", vol. 12, no. 2, pp. 52, 1991.

[10] M. M. Richter and A. Aamodt, "Case-based reasoning foundations," The Knowledge Engineering Review, vol. 20, no. 03, pp. 203-207, 2005.

[11] K. D. Althoff, R. Barletta, M. Manago, and E. Auriol, "A Review of Industrial Case-Based Reasoning Tools," AI Intelligence, Oxford, 1995.

[12] D. W. Aha, "The omnipresence of case-based reasoning in science and application," Knowledge-based systems, vol. 11, no. 5, pp. 261-273, 1998.

[13] R. Schmidt, "Case-Based Reasoning in Medicine Especially an Obituary on Lothar Gierl," Advanced Computational Intelligence Paradigms in Healthcare-1, pp. 63-87, 2007.

[14] D. B. Leake, Case-Based Reasoning: Experiences, lessons and future directions. MIT press, 1996.

[15] G. Finnie and Z. Sun, "A logical foundation for the case-based reasoning cycle," International journal of intelligent systems, vol. 18, no. 4, pp. 367-382, 2003.

[16] R. L. De Mantaras et al., "Retrieval, reuse, revision and retention in case-based reasoning," The Knowledge Engineering Review, vol. 20, no. 03, pp. 215-240, 2005.

[17] E. Simoudis and J. Miller, "Validated retrieval in case-based reasoning," in Proceedings of AAAI, pp. 310-315, 1990.

[18] D. W. Patterson, N. Rooney, and M. Galushka, "Efficient Retrieval for Case-Based Reasoning," in Proceedings of FLAIRS Conference, pp. 144-149, 2003.

[19] R. Schmidt and O. Vorobieva, "The Adaptation Problem in Medical Case-Based Reasoning Systems," Successful Case-based Reasoning Applications-I, pp. 117-141, 2010.

[20] M. Nilsson and M. Sollenborn, "Advancements and Trends in Medical Case-Based Reasoning: An Overview of Systems and System Development," in Proceedings of FLAIRS Conference, pp. 178-183, 2004.

[21] C. Pous et al., "Modeling reuse on case-based reasoning with application to breast cancer diagnosis," Artificial Intelligence: Methodology, Systems, and Applications, Lecture Notes in Computer Science, vol. 5253, pp. 322-332, 2008

[22] A. Lawanna and J. Daengdej, "Methods for case maintenance in casebased reasoning," Int. J. Comput. Inform. Eng, vol. 4, pp. 10-18, 2010.

[23] W. J. Long, "Medical informatics: reasoning methods," Artificial Intelligence in Medicine, vol. 23, no. 1, pp. 71-87, 2001.

[24] P. Lucas, "The representation of medical reasoning models in resolution-based theorem provers," Artificial intelligence in medicine, vol. 5, no. 5, pp. 395-414, 1993.

[25] R. A. Miller and A. Geissbuhler, "Diagnostic decision support systems," in Clinical Decision Support Systems, Springer New York, pp. 99-125, 2007.
[26] R. Seising, "From vagueness in medical thought to the foundations of fuzzy reasoning in medical diagnosis," Artificial Intelligence in Medicine, vol. 38, no. 3, pp. 237-256, 2006.

[27] T. R. Taylor, J. Aitchison, E. M. McGirr, "Doctors as decision-makers: a computer-assisted study of diagnosis as a cognitive skill," British medical journal, vol. 3, no. 5765, pp. 35-40, 1971.

[28] C. C. Hsu and C. S. Ho, "A hybrid case-based medical diagnosis system," in Proceedings Tenth IEEE International Conference on Tools with Artificial Intelligence, 1998, pp. 359-366, 1998.

[29] H. Eshach and H. Bitterman, "From Case-based Reasoning to Problembased Learning," Academic Medicine, vol. 78, no. 5, pp. 491-496, 2003.

[30] L. Gierl, M. Bull, and R. Schmidt, "CBR in Medicine," Case-Based Reasoning Technology, Lecture Notes in Computer Science, vol. 1400, pp. 273-297, 1998.

[31] R. T. Macura and K. Macura, "Case-based reasoning: opportunities and applications in health care," Artificial Intelligence in Medicine, vol. 9, no. 1, pp. 1-4, 1997.

[32] P. Koton, "A medical reasoning program that improves with experience," Computer methods and programs in biomedicine, vol. 30, no. 2, pp. 177-184, 1989.

[33] R. Schmidt, B. Pollwein, and L. Gierl, "Experiences with case-based reasoning methods and prototypes for medical knowledge-based systems," Artificial Intelligence in Medicine, Lecture Notes in Computer Science, vol. 1620, pp. 124-132, 1999.

[34] A. Holt, I. Bichindaritz, R. Schmidt, and P. Perner, "Medical applications in case-based reasoning," The Knowledge Engineering Review, vol. 20, no. 03, pp. 289-292, 2005.

[35] M. U. Ahmed, S. Begum, E. Olsson, N. Xiong, P. Funk, "Case-Based Reasoning for Medical and Industrial Decision Support Systems," in Successful Case-based Reasoning Applications-I, pp. 7-52, 2010.

[36] E. R. Bareiss, B. W. Porter, and C. C. Wier, "Protos: An exemplar-based learning apprentice," in Proceedings of the fourth international workshop on machine learning, pp. 12-23, August 1987.

[37] L. Gierl and S. Stengel-Rutkowski, "Integrating consultation and semiautomatic knowledge acquisition in a prototype-based architecture: Experiences with dysmorphic syndromes," Artificial Intelligence in Medicine, vol. 6, no. 1, pp. 29-49, 1994.

[38] R. T. Macura and K. J. Macura, "MacRad: Case-based retrieval system for radiology image resource," AAAI Technical Report FS-95-03, 1995.

[39] M. Haddad, D. Moertl, and G. Porenta, "SCINA: A case-based reasoning system for the interpretation of myocardial perfusion scintigrams," Computers in Cardiology, pp. 761-764, 1995.

[40] E. B. Reategui, J. A. Campbell, and B. F. Leao, "Combining a neural network with case-based reasoning in a diagnostic system," Artificial Intelligence in Medicine, vol. 9, no. 1, pp. 5-27, 1997.

[41] I. Bichindaritz, E. Kansu, and K. M. Sullivan, "Case-based reasoning in care-partner: Gathering evidence for evidence-based medical practice," Advances in case-based reasoning, Lecture Notes in Computer Science, vol. 1488, pp. 334-345, 1998.

[42] C. LeBozec, M. C. Jaulent, E. Zapletal, and P. Degoulet, "Unified modeling language and design of a case-based retrieval system in medical imaging," in Proceedings of the AMIA Symposium, pp. 887891, 1998.

[43] P. Perner, "An architecture for a CBR image segmentation system," Engineering Applications of Artificial Intelligence, vol. 12, no. 6, pp. 749-759, 1999.

[44] R. Schmidt, B. Pollwein, and L. Gierl, "Medical multiparametric time course prognoses applied to kidney function assessments," International journal of medical informatics, vol. 53, no. 2, pp. 253-263, 1999.

[45] W. Goodridge, H. Peter, and A. Abayomi, "The Case-Based Neural Network Model and its use in medical expert systems," Artificial Intelligence in Medicine, Lecture Notes in Computer Science, vol. 1620, pp. 232-236, 1999.

[46] N. H. Phuong, V. V. Thang, and K. Hirota, "Case based reasoning for medical diagnosis using fuzzy set theory," Biomedical fuzzy and human sciences: the official journal of the Biomedical Fuzzy Systems Association, vol. 5, no. 2, pp. 1-7, 2000. 
[47] C. Marling and P. Whitehouse, "Case-based reasoning in the care of Alzheimer's disease patients," Case-based reasoning research and development, Lecture Notes in Computer Science, vol. 2080, pp. 702$715,2001$.

[48] E. Golobardes, X. Llorà, M. Salamó, and J. Martí, "Computer aided diagnosis with case-based reasoning and genetic algorithms," Knowledge-Based Systems, vol. 15, no. 1, pp. 45-52, 2002.

[49] S. Montani et al., "Integrating model-based decision support in a multimodal reasoning system for managing type 1 diabetic patients," Artificial Intelligence in Medicine, vol. 29, no. 1, pp. 131-151, 2003.

[50] O. Vorobieva, L. Gierl, and R. Schmidt, "Adaptation methods in an endocrine therapy support system," in Workshop Proceedings of the Fifth International Conference on Case-Based Reasoning, NTNU, Trondheim, Norway, pp. 80-88, 2003.

[51] C. C. Hsu and C. S. Ho, "A new hybrid case-based architecture for medical diagnosis," Information Sciences, vol. 166, no. 1, pp. 231-247, 2004.

[52] M. Nilsson and P. Funk, "A case-based classification of respiratory sinus arrhythmia," Advances in Case-Based Reasoning, Lecture Notes in Computer Science, vol. 3155, pp. 673-685, 2004.

[53] M. Kwiatkowska and M. S. Atkins, "Case representation and retrieval in the diagnosis and treatment of obstructive sleep apnea: a semio-fuzzy approach," in Proceedings of the 7th European Conference on CaseBased Reasoning, pp. 25-35, 2004.

[54] P. Perner, H. Perner, S. Jänichen, and A. Bühring, "Recognition of airborne fungi spores in digital microscopic images," in Proceedings of the 17th International Conference on Pattern Recognition, 2004, vol. 3, pp. 566-569.

[55] D. Brien, J. Glasgow, and D. Munoz, "The application of a case-based reasoning system to attention-deficit hyperactivity disorder," CaseBased Reasoning Research and Development, Lecture Notes in Computer Science, vol. 3620, pp. 122-136, 2005.

[56] C. L. Chang, "Using case-based reasoning to diagnostic screening of children with developmental delay," Expert Systems with Applications, vol. 28, no. 2, pp. 237-247, 2005.

[57] W. Shi and J. A. Barnden, "Using inductive rules in medical case-based reasoning system," in MICAI 2005: Advances in Artificial Intelligence, Lecture Notes in Computer Science, vol. 3789, pp. 900-909, 2005.

[58] S. Montani and L. Portinale, "Accounting for the Temporal Dimension in Case-based Retrieval: A Framework for Medical Applications," Computational Intelligence, vol. 22, no. 3-4, pp. 208-223, 2006.

[59] M. d'Aquin, J. Lieber, J., and A. Napoli, "Adaptation knowledge acquisition: A case study for case-based decision support in oncology," Computational Intelligence, vol. 22, no. 3-4, pp. 161-176, 2006.

[60] F. Díaz, F. Fdez-Riverola, and J. M. Corchado, "GENE-CBR: A casebased reasonig tool for cancer diagnosis using microarray data sets," Computational Intelligence, vol. 22, no. 3-4, pp. 254-268, 2006.

[61] Y. J. Park, B. C. Kim, and S. H. Chun, "New knowledge extraction technique using probability for case-based reasoning: application to medical diagnosis," Expert Systems, vol. 23, no. 1, pp. 2-20, 2006.

[62] T. Töpel, J. Neumann, and R. Hofestädt, "A medical case-based reasoning component for the rare metabolic diseases database RAMEDIS," in Twentieth IEEE International Symposium on ComputerBased Medical Systems, pp. 7-11, June 2007.

[63] G. Quellec et al., "Multimedia medical case retrieval using decision trees," Proceedings of the 29th Annual International Conference of the IEEE EMBS, pp. 4536-4539, 2007.

[64] A. Cordier, B. Fuchs, J. Lieber, and A. Mille, "On-line domain knowledge management for case-based medical recommendation," in 5th workshop on CBR in the Health Sciences, a workshop of the seventh International Conference on Case-Based Reasoning (ICCBR07), pp. 285-294, 2007.

[65] C. Marling, J. Shubrook, and F. Schwartz, "Case-based decision support for patients with type 1 diabetes on insulin pump therapy," Advances in Case-Based Reasoning, Lecture Notes in Computer Science, vol. 5239, pp. 325-339, 2008.
[66] S. Little, O. Salvetti, and P. Perner, "Evaluating a Case-Based Classifier for Biomedical Applications," in 21st IEEE International Symposium on Computer-Based Medical Systems, pp. 584-586, 2008.

[67] M. U. Ahmed, S. Begum, P. Funk, N. Xiong, and B. Von Schéele, "Case-based reasoning for diagnosis of stress using enhanced cosine and fuzzy similarity," Transactions on Case-Based Reasoning for Multimedia Data, vol. 1, no. 1, pp. 3-19, 2008.

[68] Y. Rodríguez et al., "Prediction of Pediatric Risk Using a Hybrid Model Based on Soft Computing Techniques," in MICAI 2008: Advances in Artificial Intelligence, pp. 472-481, 2008.

[69] J. M. Corchado, J. Bajo, and A. Abraham, "GerAmi: Improving healthcare delivery in geriatric residences," Intelligent Systems, vol. 23, no. 2, pp. 19-25, 2008.

[70] J. F. De Paz, S. Rodríguez, J. Bajo, and J. M. Corchado, "Case-based reasoning as a decision support system for cancer diagnosis: A case study," International Journal of Hybrid Intelligent Systems, vol. 6, no. 2, pp. 97-110, 2009.

[71] O. U. Obot and F. M. E. Uzoka, "A framework for application of neurocase-rule base hybridization in medical diagnosis," Applied Soft Computing, vol. 9, no. 1, pp. 245-253, 2009.

[72] R. H. Lin, "An intelligent model for liver disease diagnosis," Artificial Intelligence in Medicine, vol. 47, no. 1, pp. 53-62, 2009.

[73] H. Ahn and K. J. Kim, "Global optimization of case-based reasoning for breast cytology diagnosis," Expert Systems with Applications, vol. 36, no. 1, pp. 724-734, 2009.

[74] S. Begum, M. U. Ahmed, P. Funk, N. Xiong, and B. Von Schéele, “A case-based decision support system for individual stress diagnosis using fuzzy similarity matching," Computational Intelligence, vol. 25, no. 3, pp. 180-195, 2009.

[75] B. Yao and S. Li, "ANMM4CBR: a case-based reasoning method for gene expression data classification," Algorithms for Molecular Biology, vol. 5, no. 1, pp. 5-14, 2010.

[76] D. X. Gu, C. Y. Liang, X. G. Li, S. L. Yang, and P. Zhang, "Intelligent technique for knowledge reuse of dental medical records based on casebased reasoning," Journal of medical systems, vol. 34, no. 2, pp. 213222, 2010.

[77] R. H. Lin and C. L. Chuang, "A hybrid diagnosis model for determining the types of the liver disease," Computers in Biology and Medicine, vol. 40, no. 7, pp. 665-670, 2010.

[78] R. Jagannathan, S. Petrovic, A. McKenna, and L. Newton, "A fuzzy non-linear similarity measure for case-based reasoning systems for radiotherapy treatment planning," in Artificial Intelligence Applications and Innovations, IFIP Advances in Information and Communication Technology, vol. 339, pp. 112-119, 2010.

[79] M. U. Ahmed, S. Begum, P. Funk, N. Xiong, and B. Von Schéele, “A multi-module case-based biofeedback system for stress treatment," Artificial Intelligence in Medicine, vol. 51, no. 2, pp. 107115, 2011.

[80] N. Douali, J. De Roo, E. I. Papageorgiou, and M. C. Jaulent, "Case Based Fuzzy Cognitive Maps (CBFCM): new method for medical reasoning: comparison study between CBFCM/FCM," in 2011 IEEE International Conference on Fuzzy Systems (FUZZ), pp. 844-850, 2011.

[81] C. L. Chuang, "Case-based reasoning support for liver disease diagnosis," Artificial intelligence in medicine, vol. 53, no. 1, pp. 15-23, 2011.

[82] S. Petrovic, N. Mishra, and S. Sundar, "A novel case based reasoning approach to radiotherapy planning," Expert Systems with Applications, vol. 38, no. 9, pp. 10759-10769, 2011.

[83] M. van den Branden, N. Wiratunga, D. Burton, and S. Craw, "Integrating case-based reasoning with an electronic patient record system," Artificial Intelligence in Medicine, vol. 51, no. 2, pp. 117-123, 2011.

[84] B. López et al., "eXiT* CBR: A framework for case-based medical diagnosis development and experimentation," Artificial intelligence in medicine, vol. 51, no. 2, pp. 81-91, 2011.

[85] B. López et al., "System overview: Breast cancer prognosis through CBR," CBR in the Health Sciences, Workshop at the Twentieth 
International Conference on Case-Based Reasoning (ICCBR 2012), pp. 105-110, 2012.

[86] M. U. Ahmed, S. Begum, and P. Funk, "A hybrid case-based system in clinical diagnosis and treatment," in 2012 IEEE-EMBS International Conference on Biomedical and Health Informatics (BHI), pp. 699-704, 2012.

[87] S. Montani, G. Leonardi, A. Bottrighi, L. Portinale, and P. Terenziani, "Extending a time series retrieval tool to deal with sub-series matching: an application to the hemodialysis domain," in CBR in the Health Sciences, Workshop at the Twentieth International Conference on CaseBased Reasoning (ICCBR 2012), pp. 59-68, 2012.

[88] A. Khelassi, and M. A. Chikh, "Cognitive Amalgam with a Fuzzy sets and case based reasoning for accurate cardiac arrhythmias diagnosis," in CBR in the Health Sciences, Workshop at the Twentieth International Conference on Case-Based Reasoning (ICCBR 2012), pp. 69-79, 2012.

[89] C. Marling, R. Bunescu, J. Shubrook, and F. Schwartz, "System Overview: The 4 Diabetes Support System," in CBR in the Health Sciences, Workshop at the Twentieth International Conference on CaseBased Reasoning (ICCBR 2012), pp. 81-86, 2012.

[90] J. M. Juarez et al., "Supporting Frontotemporal Dementia Protocols by CBR Graph Models," in Workshop Proceedings of the 20th International Conference on Case-Based Reasoning (ICCBR 2012), pp. 87-99, 2012.

[91] M. U. Ahmed, S. Begum, and P. Funk, "System Overview on the Clinical Decision Support System for Stress Management," in Proceedings of the ICCBR 2012 Workshop on CBR in the Health Sciences, pp. 111-116, 2012.

[92] M. U. Ahmed, A. M. Islam, and A. Loutfi, "A case-based patient identification system using pulseoximeter and a personalized health profile," in Proceedings of the ICCBR 2012 Workshop on CBR in the Health Sciences, pp. 117-127, 2012.

[93] S. Begum, M. U. Ahmed, and S. Barua, "Multi-scale entropy analysis and case-based reasoning to classify physiological sensor signals," in Proceedings of the ICCBR 2012 Workshop on CBR in the Health Sciences, pp. 129-138, 2012.

[94] V. E. Ekong, U. G. Inyang, and E. A. Onibere, "Intelligent decision support system for depression diagnosis based on neuro-fuzzy-CBR hybrid," Modern Applied Science, vol. 6, no. 7, pp. 79-88, 2012.

[95] M. L. Huang, Y. H. Hung, W. M. Lee, R. K. Li, and T. H. Wang, "Usage of case-based reasoning, neural network and adaptive neurofuzzy inference system classification techniques in breast cancer dataset classification diagnosis," Journal of medical systems, vol. 36, no. 2, pp. 407-414, 2012.

[96] S. Montani, G. Leonardi, S. Ghignone, and L. Lanfranco, "Flexible casebased retrieval for comparative genomics," Applied intelligence, vol. 39, no. 1, pp. 144-152, 2013.

[97] S. Chattopadhyay, S. Banerjee, F. A. Rabhi, and U. R. Acharya, "A Case-Based Reasoning system for complex medical diagnosis," Expert Systems, vol. 30, no. 1, pp. 12-20, 2013.

[98] A. Pla, B. LóPez, P. Gay, and C. Pous, "eXiT* CBR. v2: Distributed case-based reasoning tool for medical prognosis," Decision Support Systems, vol. 54, no. 3, pp. 1499-1510, 2013.
[99] Y. Leal et al., "Principal component analysis in combination with casebased reasoning for detecting therapeutically correct and incorrect measurements in continuous glucose monitoring systems," Biomedical Signal Processing and Control, vol. 8, no. 6, pp. 603-614, 2013.

[100]D. Teodorović, M. Šelmić, and L. Mijatović-Teodorović, "Combining case-based reasoning with Bee Colony Optimization for dose planning in well differentiated thyroid cancer treatment," Expert Systems with Applications, vol. 40, no. 6, pp. 2147-2155, 2013.

[101]J. Henriet, P. E. Leni, R. Laurent, and M. Salomon, "Case-Based Reasoning adaptation of numerical representations of human organs by interpolation," Expert Systems with Applications, vol. 41, no. 2, pp. 260-266, 2014.

[102]D. A. Sharaf-El-Deen, I. F. Moawad, and M. E. Khalifa, "A new hybrid case-based reasoning approach for medical diagnosis systems," Journal of medical systems, vol. 38, no. 2, pp. 1-11, 2014.

[103]Z. Yin et al., "A clinical decision support system for the diagnosis of probable migraine and probable tension-type headache based on casebased reasoning," The journal of headache and pain, vol. 16, no. 1, pp. 29, 2015.

[104]A. Tyagi and P. Singh, "ACS: Asthma Care Services with the Help of Case Base Reasoning Technique," Procedia Computer Science, vol. 48, pp. 561-567, 2015.

[105]G. Khussainova, S. Petrovic, and R. Jagannathan, "Retrieval with Clustering in a Case-Based Reasoning System for Radiotherapy Treatment Planning, 2015. (http://dx.doi.org/10.1088/17426596/616/1/012013).

[106]R. M. Saraiva, J. Bezerra, M. Perkusich, H. Almeida, and C. Siebra, “A Hybrid Approach Using Case-Based Reasoning and Rule-Based Reasoning to Support Cancer Diagnosis: A Pilot Study," Studies in health technology and informatics, vol. 216, pp. 862-866, 2015.

[107]S. Chakraborty, C. Pal, S. Chatterjee, B. Chakraborty, and N. Ghoshal, "Knowledge-Based System Architecture on CBR for Detection of Cholera Disease," Intelligent Computing and Applications, Advances in Intelligent Systems and Computing, vol. 343, pp. 155-165, 2015.

[108]S. Nasiri, J. Zenkert, and M. Fathi, "A Medical Case-Based Reasoning Approach Using Image Classification and Text Information for Recommendation," Advances in Computational Intelligence, Lecture Notes in Computer Science, vol. 9095, pp. 43-55, 2015.

[109]S. Banerjee and A. R. Chowdhury, "Case Based Reasoning in the Detection of Retinal Abnormalities Using Decision Trees," Procedia Computer Science, vol. 46, pp. 402-408, 2015.

[110]X. Blanco, S. Rodríguez, J. M. Corchado, and C. Zato, "Case-based reasoning applied to medical diagnosis and treatment," Distributed Computing and Artificial Intelligence, Advances in Intelligent Systems and Computing, vol. 217, pp. 137-146, 2013.

[111]I. Bichindaritz, "Solving safety implications in a case based decisionsupport system in medicine," in Proceedings of the Fifth International Conference on Case-based Reasoning Workshop on Case-Based Reasoning in the Health Sciences, pp. 178-183, 2003. 